\title{
Evaluating the efficacy of a low-cost, minimally- invasive cognitive MRI targeted biopsy protocol in the Prostate Imaging Reporting and Data System (PI-RADS) version 2 era.
}

Yuta Takeshima ( $\square$ yutatake@hotmail.com )

Tokyo Daigaku Ikagaku Kenkyujo https://orcid.org/0000-0002-1195-6484

Yoshinori Tanaka

Musashino Red Cross Hospital

Kotaro Takemura

University of Tokyo

Shusaku Nakazono

Musashino Red Cross Hospital

Eiko Yamashita

Musashino Red Cross Hospital

Haruki Kume

University of Tokyo

Research article

Keywords: prostate cancer, multiparametric magnetic resonance imaging, prostate imaging reporting and data system, cognitive targeting biopsy, prostate specific antigen density

Posted Date: December 31st, 2019

DOl: https://doi.org/10.21203/rs.2.19771/v1

License: (c) (i) This work is licensed under a Creative Commons Attribution 4.0 International License. Read Full License 


\section{Abstract}

Background: New MRI-guided targeting biopsy methods have increased cancer yield of prostate biopsies. However, cost and time constraints have made it difficult for many institutions to implement these newer methods. We evaluated the diagnostic performance of a low-cost, minimally-invasive, cognitive MRItargeted biopsy protocol based on 1.5T multiparametric MRI graded with Prostate Imaging Reporting and Data System version 2 that is easily implemented in any low- to intermediate- volume center.

Methods: Retrospective analysis of 255 patients who underwent prostate biopsy between December 2016 and March 2019 at a single facility. Indication for biopsy was based on clinical parameters including 1.5T multiparametric MRI. In addition to 10-core systematic biopsy, targeted cores were obtained with cognitive recognition under ultrasound. A control group of 198 patients biopsied without prior MRI from January to December 2015 was also analyzed.

Results: Prostate biopsy preceded by MRI had a significantly higher probability of detecting both prostate cancer $(68.1 \%$ vs. $43.6 \%)$ and clinically significant cancer $(56.2 \%$ vs. $29.4 \%)$ ( $p$ values< 0.01$)$.

Combination of systematic biopsy and targeted biopsy outperformed either regimen alone for detection of prostate cancer. Multivariate analysis showed PSA density and prostate imaging reporting and data system score were independent risk factors of prostate cancer. A proposed diagnostic model showed sensitivity of $88.6 \%$, specificity of $55 \%$, PPV of $81.2 \%$, NPV of $68.8 \%$, and accuracy of $78 \%$. Prostate imaging reporting and data system score was correlated with a higher presence of prostate cancer, clinically significant prostate cancer, and a higher pathological grade.

Conclusions: Incorporation of pre-biopsy MRI imaging, scoring, and targeted biopsy improved cancer yield and achieved diagnostic performance comparable to newer methods of higher cost. Future alterations of possible benefit included increasing the number of target cores per lesion, and combining prostate imaging reporting and data system score and PSA density as indicators for biopsy.

\section{Background}

Systematic transrectal ultrasound (TRUS)- guided biopsy has long been the standard for diagnosis of prostate cancer. However, systematic biopsy (SB) has a fairly high percentage of false-negative results at $21-34 \%^{1,2}$, and overdiagnosis of clinically-insignificant cancer has been mentioned as a limitation ${ }^{3}$.

Furthermore, biopsies are associated with risks of bleeding, infection, and voiding difficulty ${ }^{4}$. Indications for a prostate biopsy are elevated prostate-specific antigen (PSA) levels, positive digital rectal examination (DRE), hypo-echoic lesions on TRUS, and more recently, detection of lesions on multiparametric magnetic resonance imaging (mpMRI). Recent studies have shown high sensitivity and specificity for mpMRI in diagnosing clinically significant prostate cancer (csPCa) ${ }^{5}$, and conducting mpMRI before biopsy has been shown to reduce unnecessary biopsies ${ }^{6}$. It was further popularized by the introduction of Prostate Imaging Reporting and Data System (PI-RADS) ${ }^{7}$ in 2012. Updated in 2015, PIRADS version2 provided a five-point scale of reference for urologists as indications for prostate biopsy ${ }^{8}$. 
Increasing evidence on the efficacy of mpMRI to diagnose csPCa has led to several MRI-guided targeted biopsy (TB) protocols, each of which have delivered promising results: cognitive-targeting biopsy (COGTB) for which visual registration is used to determine the point of biopsy on TRUS ${ }^{9}$, in-bore MRI-targeting biopsy (MRI-TB) performed within the MRI suite with MR-imaging compatible software ${ }^{10}$, and MRI/US fusion targeting biopsy (FUS-TB) in which MR and TRUS images are fused for real-time visual feedback ${ }^{11}$. However, the high cost and time constraints of FUS-TB and MRI-TB have made it difficult for many hospitals to implement these newer methods while maintaining capital regarding prostate biopsy. In line with the evidence and circumstances, we adopted a protocol we believe to be cost-effective and minimally-invasive, easily implemented in any low- to intermediate-volume center. Following 1.5T mpMRI of the prostate and evaluation with PI-RADS v2, patients received a COG-TB of 1-2 cores per reported lesion plus concomitant 10-core SB under local anesthesia on an outpatient basis. We will evaluate the outcomes of the first two years of implementing this protocol and report its effect on prostate cancer detection.

\section{Methods}

Patient population

All data was collected retrospectively from electronic patient records with approval of the institutional review board of the Institute of Medical Science, University of Tokyo (Approval No.2019-2-0607), and Musashino Red Cross Hospital (Approval No.1025). From December 2016 to March 2019, 267 patients with elevated PSA underwent mpMRI and subsequently received prostate biopsy at our institution. Twelve patients were excluded for incomplete mpMRI scans, incomplete PI-RADs evaluation, and in the case of patients with PI-RADS score $\geq 3$, no target cores acquired. To compare biopsies before and after the introduction of $\mathrm{mPMRI}$ and TB, we extracted data for a control group of 198 patients who received prostate biopsy without prior mpMRI or TB from January to December 2015.

MRI

All imaging studies were performed with a 1.5T MRI scanner (Signa HDxt 1.5T, GE Healthcare, Chicago, IL), using 8 channel body array coils. Endorectal coil was not used. Sequences obtained included T2 weighted imaging (axial, coronal, and sagittal planes, $3 \mathrm{~mm}$ thickness), diffusion-weighted imaging (bvalue 2000), and dynamic contrast-enhanced imaging with $15 \mathrm{ml}$ intravenous gadolinium. Classification of mpMRI findings were conducted by a single experienced radiologist according to PIRADS v2.

Prostate biopsy

All DRE, TRUS, and biopsies were performed by six experienced urologists. Patients with lesions of PIRADS score 1-2 underwent a routine 10-core SB. Patients with lesions of PI-RADS score 3 received both TB and SB. One or two TB cores were taken from each suspicious lesion. For SB, a 10-core biopsy of lateral cores from the basal, mid-gland and apical sections and mid-lobar cores from the basal and apical 
sections was conducted. Biopsies were performed using a Toshiba Xario100 ultrasound system (Canon Medical Systems, Tochigi, Japan) under local anesthesia with $8 \mathrm{ml}$ of $1 \%$ lidocaine, with no hospitalization.

\section{Pathology}

All biopsy specimens were embedded, fixed, and underwent histopathological analysis by experienced pathologists according to International Society of Urological Pathology (ISUP) standards. A Gleason Score was given for each core, and an overall ISUP score was determined. CsPCa was defined as ISUP score 2 for this study.

Statistical analysis

We evaluated the following factors for each patient: age, initial PSA, free/total PSA ratio (F/T), prostate volume (PV), PSA density (PSAD), DRE and TRUS findings, mpMRI findings including PI-RADS score for each detected lesion, previous biopsy history, and biopsy results. Independent sample t tests were performed to compare variables between PCa-positive and -negative groups, and also csPCa-positive and -negative groups. Detection rates of TB and SB were analyzed with McNemar's chi-squared test. Multivariate analyses were performed with logistic regression analysis to determine significant predictors of PCa and csPCa. To determine cutoff value for continuous and ordinal variables such as PSAD and PIRADS score, we used receiver operating characteristic (ROC) curve and Youden index. Comparison of ROC curves were conducted with DeLong's test. Diagnostic performance was evaluated using sensitivity, specificity, positive predictive value (PPV), negative predictive value (NPV), and accuracy, which were reported with $95 \%$ Confidence interval $(\mathrm{Cl})$. Correlation of ordinal variables such as ISUP score and PIRADS score was determined using Spearman's rank correlation coefficient, or " $r$ ".

All statistical analyses were performed by using software (JMP Pro, version 14.2, SAS, Cary, NC), and a $p$ value of $<0.05$ was considered to indicate statistical significance.

\section{Results}

Patient demographics and biopsy results are shown in Table 1. For the purposes of this analysis, the older cohort which received SB without mpMRI was labeled "CP" (conventional protocol), and the newer cohort which received both SB and TB preceded by mpMRI was labeled "RP" (revised protocol). We found a tendency for patients with high PSA levels to forgo pre-biopsy mpMRI in favor of a swift diagnosis with immediate prostate biopsy, and significantly more patients with a PSA level over $50 \mathrm{ng} / \mathrm{ml}$ were included for the CP cohort (24 of 187 patients, range $50.8-1428.7 \mathrm{ng} / \mathrm{ml}$ ) than the RP cohort (4 of 255 patients, range 50.4-63.5) ( $p$ value<0.01). In order to conform the cohorts for direct comparison, we excluded these patients for a total of 251 and 163 patients for this analysis only. As a result, no difference was seen between the two groups in age, PSA, PV, PSAD, TRUS-findings, or previous prostate biopsies. F/T and DRE findings were significantly more speculative of prostate cancer in the CP cohort. Despite this, the RP 
cohort showed a significantly higher probability of detecting both prostate cancer $(68.1 \%$ vs. $43.6 \%, p$ value $<0.001)$ and clinically significant cancer $(56.2 \%$ vs. $29.4 \%$, p value $<0.001)$.

We analyzed how SB and TB fared individually with biopsy results as standard of reference (Table 2). Both SB and TB each accounted for over $70 \%$ of total prostate cancer cases, but the combination of SB and TB statistically outperformed SB alone and TB alone for the detection of both PCa and csPCa. SB alone outperformed TB alone for the detection of both $\mathrm{PCa}$ and csPCa in our cohort. The number of cores biopsied per suspicious lesion showed no correlation with detection of either PCa ( $r=0.1690)$ or csPCa ( $r=0.1754)$. Overall, 201 of 435 total TB cores $(46.2 \%)$ and 423 of 2810 total SB cores $(15.1 \%)$ were positive for PCa.

The RP cohort was analyzed for risk factors of PCa and csPCa (Table3). Cutoffs were determined by Youden Index and rounded to the nearest round number with the largest AUC. Univariate analysis showed age, PSA, PV, PSAD, positive DRE and TRUS findings, and PI-RADS score to be a significant risk factor of both PCa and csPCa. As PSA, PV, and PSAD are obviously heavily correlated, we conducted ROC curve analysis, and found AUC for PSAD to be greatest both as nominal variables and continuous variables (Suppl. Table 1). We determined that PSAD was the strongest predictor of PCa and csPCa, and thus most suitable for multivariate analysis. Multivariate analysis showed that age, PSAD, and PI-RADS score were independent risk factors for detection of PCa, and PSAD and PI-RADS score were independent risk factors for detection of csPCa.

Since excluding patients of younger age for biopsy would be counterproductive due to longer life expectancy and larger benefit from radical treatment, we examined the two remaining risk factors to determine whether their combination would result in better risk evaluation. The combination of ROC curves are shown in Fig.1. For both PCa and csPCa, AUC was significantly greater for the combination of PSAD and PI-RADS score than PSAD or PI-RADS score alone.

Diagnostic performance was evaluated for PSA, PV, PSAD, PI-RADS score, and the combination of PIRADS and PSAD. The same cutoff values as those given in Table 3 were used. For the combination model, we proposed a model based on the ROC curve above where one or more risk factors above the cutoff point (PSAD>0.25, PI-RADS score4) would be considered positive (Model 1). When applying to patient selection in a real-world setting, a stringent cutoff value would lead to more significant cancers being left undiagnosed. Therefore, we adopted for comparison a second combination model with lower cutoff values proposed in past literature, in which all patients with PI-RADS score above 4, and those with PI-RADS score 3 with PSAD above 0.15 would be considered positive (Model 2). Results are listed on Table 4. Cutoff of PI-RADS score at $\geq 3$ resulted in high sensitivity at $98.3 \%$ but low specificity of $10 \%$, which led to Model 2 having a similarly low specificity of $23.8 \%$. Model 1 resulted in a higher specificity (55\%), PPV(81.2\%), NPV(68.8\%), and overall accuracy(78\%), with minimal dropoff in sensitivity(88.6\%).

The pathological results of prostate biopsy are shown in Fig. 2, stratified by PI-RADs scores. The ratio of positive biopsies can be seen rising along with the PI-RADS score $(27.2 \%$ vs. $39.4 \%$ vs. $77.5 \%$ vs. 91.4 , p value $<0.0001)$, and a correlation was seen between PCa detection and PI-RADS score $(r=0.44)$. A 
correlation was also seen between csPCa detection and PI-RADS score $(r=0.45)$. ISUP score also tends to be higher in patients with higher PI-RADS scores $(r=0.47)$.

\section{Discussion}

Introduction of pre-biopsy mpMRI and PI-RADS scoring along with MRI-targeting have improved prostate biopsy. However, while FUS-TB generally achieved the highest detection rate in past studies, others reported that COG-TB showed no significant difference in cancer detection on a per patient basis ${ }^{12-14}$. Furthermore, multiple studies have pointed out a decrease in cost-effectiveness with MRI-TB and FUS$\mathrm{TB}^{15-17}$. MRI-TB, while having the most direct targeting of the specified lesion, is associated with longer operative time, increased costs, and patient discomfort. Inability to perform concomitant systematic biopsy has also been noted as a demerit ${ }^{10}$. FUS-TB, while delivering promising results, currently does not have a billable medical care code in our country, and additional cost of equipment is not compensated for. As of 2018, the Ministry of Health, Labor, and Welfare has not deemed the evidence sufficient to provide for the method under public health insurance. Our institution is one of many that have been unable to incorporate FUS-TB for this reason. In addition, our MRI scanner is 1.5T, inferior in resolution to the newer-generation $3 \mathrm{~T}$ models. Although PI-RADS scoring has allowed urologists to incorporate mpMRI into patient selection for prostate biopsy far more easily, it was unclear whether pre-biopsy mpMRI would be beneficial under these circumstances. We conducted this retrospective study to analyze whether conducting 1.5T mpMRI imaging before biopsy and adding cognitive-targeted biopsy to systematic biopsy in a minimally-invasive protocol improved diagnostic yield, and to determine what measures we could take to further improve within the constraints of cost.

Conducting mpMRI before biopsy significantly improved the percentage of positive biopsies for both PCa and cSPCa, consistent with previous reports comparing SB and TB. Past studies have compared COG-TB and SB cores in a single cohort and reported that the combination of COG-TB and SB was superior to SB or COG-TB alone ${ }^{18}$. Several groups have also applied this to FUS-TB and found the combination of SB and FUS-TB to be superior, especially in the detection of csPCa ${ }^{19-21}$. In all studies except the PI-RADS score3 subgroup in Hansen et al. ${ }^{21}$, TB alone showed a tendency to outperform SB alone. Although comparison between cohorts may be difficult, cancer detection rate for TB alone was $52.9 \%$ for all PCa, similar to those previously reported for COG-TB (36-59\%) ${ }^{13,14,18,22}$ and FUS-TB (36-77\%) ${ }^{13,18,19,22}$. It is of note that SB alone outperformed TB alone in our cohort. $46.2 \%$ of TB cores were positive for PCa, similar to past reports (32-47\%) ${ }^{12-13}$, and approximately 3 times more likely than the SB core to be PCa-positive, so this difference most likely comes from the number of TB cores per biopsy. For comparison, the average number of COG-TB cores per patient in our study was 1.7, as opposed to 4 for Peuch et al. ${ }^{12}$ and 7 for Borkowetz et al. ${ }^{18}$. We failed to detect a correlation between the number of TB cores per lesion and cancer detection, probably due to small sample size with 2 or more cores taken per lesion in only 10 cases $(4.1 \%)$. Although a single study reports that a second TB core per lesion adds minimal value in $\mathrm{MRI}-\mathrm{TB}^{23}$, a statement from the American Urological Association recommends a minimum of two biopsy cores per suspicious lesion ${ }^{24}$. In prioritizing minimal invasiveness we initially adopted a one TB core per 
lesion protocol, but it seems likely that more TB cores per lesion would lead to higher accuracy moving forward.

Logistic regression analysis identified PI-RADS score and PSAD as independent predictors of csPCa. A few reports have incorporated ROC curve analysis to support this conclusion ${ }^{25-27}$. In these studies ROC for PSAD was presented as a continuous variable, but in a real-life setting, decision making for recommendation of biopsy would be better served with a designated cutoff value. We used Youden index to determine cutoffs and constructed a model that would be easy to implement in everyday practice; i.e., recommending biopsy to patients with either PSAD0.25 or PI-RADS score4. Venderlink et al. ${ }^{25}$ proposed a similar model in which a PSAD cut-off of 0.15 was proposed for patients with PI-RADS score3. Both diagnostic models showed high sensitivity and accuracy in our cohort, although specificity and NPV was higher in our model. NPV and specificity were generally low across all parameters, probably due to the low percentage of PI-RADS score 1-2 (11 of 255 patients, 4.3\%) which decreased the true negative population. The value of the tradeoff between reducing biopsies by $23.1 \%$ while missing $9.7 \%$ of csPCa is debatable, but we feel the easy-to-implement model would be a feasible option going forward.

Interestingly, in the sole report which states that PSAD did not improve PCa detection ${ }^{28}$, the ROC curve for PI-RADS score had an extremely high AUC of 0.92. A learning curve for PI-RADS evaluation has been detected in multiple studies, the ratio of score3 lesions progressively decreasing along with radiologist experience. This may indicate that modeling with variables such as PSAD would have less value in the future with the refinement of MRI evaluation.

Lastly, we evaluated the performance of MRI prostate imaging. Thompson et al. reported that magnet strength (1.5T vs. $3 \mathrm{~T}$ ) was not an independent predictor of csPCa detection ${ }^{29}$. While imaging resolution is obviously higher with $3 \mathrm{~T} \mathrm{MRI}$, there may not be as much of a difference in the quality of its evaluation. In our cohort, PI-RADS was correlated with a higher presence of PCa, csPCa, and a higher ISUP score. These findings were consistent with previous studies using either $1.5 \mathrm{~T}^{19}$ or $3 \mathrm{~T}^{20}$. With our results reflecting traits from existing literature, we concluded that PI-RADS v2 scoring of 1.5MRI at our institution was of a comparable quality to scoring with newer methods of imaging.

This study is limited by its retrospective nature, especially by patient selection bias which is apparent in the small number of PI-RADS score 1-2. This directly led to lower specificity and NPV for diagnostic models. However, since this is a result of less patients with low PI-RADS score electing for biopsy, we feel it reflects real-life clinical practice. Multiple urologists were involved with patient selection and biopsy procedure, possibly leading to differing standards in recommending pre-biopsy MRI or levels of biopsy technique. We also acknowledge that defining csPCa as ISUP score $\geq 2$ regardless of core cancer volume is debatable. Unfortunately, core cancer volume is not currently calculated for all cores at our institution, therefore we were unable to incorporate it into our classifications.

\section{Conclusion}


In summary, we found that incorporation of pre-biopsy 1.5T mpMRI imaging, PI-RADS v2 scoring, and a minimally-invasive combined SB and COG-TB protocol improved prostate cancer yield and achieved diagnostic performance comparable to newer methods with higher cost. Future alterations that may be beneficial included obtaining more target cores per suspicious lesion, and incorporating the combination of PIRADS v2 score and PSA density as indicators for prostate biopsy. Along with the original protocol, both alterations are easily applicable in everyday clinical practice regardless of the scale of the institution.

\section{Declarations}

Ethics approval and consent to participate: All data was collected retrospectively from electronic patient records with approval of the institutional review board of the Institute of Medical Science, University of Tokyo (Approval No.2019-2-0607), and Musashino Red Cross Hospital (Approval No.1025).

Consent for Publication: Not applicable

Availability of data and materials: The datasets used and/or analysed during the current study are available from the corresponding author on reasonable request.

Competing Interests: None declared.

Funding: None declared.

Authors' Contributions: YuT was responsible for conception and design of the study, as well as acquisition and analysis of data, and drafted the manuscript. YoT oversaw the conception and design of the study, and supervised data acquisition and drafting of the manuscript. KT and SN contributed equally to $\mathrm{YT}$ in the acquisition of data. EY served a primary role in interpretation of the data. HK provided supervision for the drafting of the manuscript, and a chief role in critical revision of the manuscript. All authors read and approved the final manuscript.

Acknowledgements: Not applicable.

\section{Abbreviations}

$\mathrm{AUC}=$ area under curve

$\mathrm{Cl}=$ confidence interval

COG-TB=cognitive targeting biopsy

$\mathrm{CP}=$ conventional protocol

csPCa=clinically significant prostate cancer 
DRE=digital rectal examination

$F / T=$ free $P S A /$ total $P S A$ ratio

FUS-TB=MRI/US fusion targeting biopsy

ISUP=International Society of Urological Pathology

$\mathrm{mpMRI}=$ multiparametric magnetic resonance imaging

MRI-TB=in-bore MRI targeting biopsy

NPV=negative predictive value

$\mathrm{OR}=$ odds ratio

$\mathrm{PCa}=$ prostate cancer

PI-RADS = prostate imaging reporting and data system

PPV= positive predictive value

PSA=prostate specific antigen

$P S A D=P S A$ density

$\mathrm{PV}=$ prostate volume

$\mathrm{ROC}=$ receiver operating characteristic

$\mathrm{RP}=$ revised protocol

$\mathrm{SB}=$ systematic biopsy

TB=targeted biopsy

TRUS=transrectal ultrasound

\section{References}

1. Singh H, Canto L, Shariat SF et al. Predictors of prostate cancer after initial negative systematic 12 core biopsy. J.Urol. 2004; 171:1850-4

2. Keetch DW, Catalona WJ, Smith DS. Serial prostatic biopsies in men with persistently elevated serum prostate specific antigen values. J.Urol. 1994; 151: 1571-4

3. Draisma G, Etxioni R, Tsodikov A et al. Lead time and overdiagnosis in protate-specific antigen screening: importance of methods and context. J.Natl.Cancer.Inst. 2009; 101: 374-383 
4. Loeb S, Vellekoop A, Ahmed HU et al. Systematic review of complications of prostate biopsy. Eur. Urol. 2013; 64: 876-92

5. Hoeks CM, Barentsz JO, Hambrock T et al. Prostate cancer: multiparametric MR imaging for detection, localization, and staging. Radiology 2011; 261: 46-66

6. An JY, Sidana A, Holzman SA et al. Ruling out clinically significant prostate cancer with negative multi-parametric MRI. Int. Urol. Neph. 2018; 50: 7-12

7. Barentsz JO, Richenburg J, Clements R et al. ESUR prostate MR guidelines 2012. Eur. Radiol. 2012; 22:746-57

8. Barentsz JO, Weinreb JC, Verma S et al. Synopsis of the PI-RADS v2 guidelines for multiparametric prostate magnetic resonance imaging and recommendations for use. Eur. Urol. 2016; 69: 41-9

9. Peuch P, Ouzzane A, Gailard V et al. Multiparametric MRI-Targeted TRUS prostate biopsies using visual registration. Biomed. Res. Int. 2014; doi: 10.1155/2014/819360

10. Schiavina R, Vagnoni V, D'Agostino D et al. "In-bore" MRI-guided prostate biopsy using an endorectal nonmagnetic device: a prospective study of 70 consecutive patients. Clin. Genitourin. Cancer 2017; 15: 417-427

11. Hakozaki $Y$, Matsushima $H$, Kumagai J et al. A prospective study of magnetic resonance imaging and ultrasonography (MRI/US)-fusion targeted biopsy and concurrent systematic transperineal biopsy with the average of 18-cores to detect clinically significant prostate cancer. BMC Urol. 2017; 17: 117

12. Peuch $P$, Rouviere $O$, Renard-Penna R et al. Multiparametric MR-targeted Biopsy with Cognitive and transrectal US-MR fusion Guidance versus systematic biopsy- Prospective multicenter study. Radiology 2013; 268: 461-469

13. Wysock JS, Rosenkratz AB, Huang WC et al. A prospective, blinded comparison of magnetic resonance (MR) imaging-ultrasound fusion and visual estimation in the performance of MR-targeted prostate biopsy: The PROFUS Trial. Eur. Urol. 2014; 66: 343-351

14. Osses DF, van Asten JJ, Tijsterman JD. Cognitive-targeetd versus magnetic resonance Imaging Guided prostate biopsy in prostate cancer detection. Curr. Urol. 2017; 11: 182-188

15. Pahwa S, Schiltz NK, Ponsky LE, Lu Z, Griswold MA, Gulani V. Cost-effectiveness of MR Imagingguided strategies for detection of prostate cancer in biopsy-naïve men. Radiology 2017; 285; 157-66

16. Altok M, Kim B, Patel BB et al. Cost and efficacy comparison of five prostate biopsy modalities: a platform for integrating cost into novel-platform comparative research. Prostate Cancer Prostatic Dis. 2018 ; 21 : 524-532

17. Hutchinson RC, Costa DN, Lotan Y. The economic effect of using magnetic resonance imaging and magnetic resonance ultrasound fusion biopsy for prostate cancer diagnosis. Urol. Oncol. 2016; 34: 296-302

18. Borkowetz A, Platzek I, Toma M et al. Evaluation of prostate imaging reporting and data classification in the prediction of tumor aggressiveness in targeted magnetic resonance/ultrasoundfusion biopsy. Urol. Int. 2017; 99: 177-185

Page $10 / 13$ 
19. Maxeiner A, Kittner B, Blobel C et al. Primary magnetic resonance imaging/ultrasonography fusionguided biopsy of the prostate. BJU Int. 2018; 122: 211-218

20. Mannaerts CK, Kajtazovic A, Lodeizen OAP et al. The added value of systematic biopsy in men with suspicion of prostate cancer undergoing multiparametric MRI-targeted biopsy. Urol. Oncol. 2019; 37: 298 e1.-e9

21. Hansen NL, Kesch C, Barrett T et al. Multicentre evauation of targeted and systematic biopsies using magnetic resonance and ultrasound image-fusion guided transperineal prostate biopsy in patients with a previous negative biopsy. BJU Int. 2017; 120: 631-638

22. Delongchamps NB, Peyromaure $M$, Schull $A$ et al. Pre-biopsy magnetic resonance imaging and prostate cancer detection: comparison of random and targeted biopsies. J. Urol. 2013; 189: 493-499

23. Schimmoller L, Quentin M, Blondin D et al. Targeted MRI-guided prostate biopsy: are two biopsy cores per MRI-lesion required? Eur. Radiol. 2016; 26: 3858-3864

24. Rosenkratz $A B$, Verma $S$, Choyke $P$ et al., Prostate magnetic resonance imaging and magnetic resonance imaging targeted biopsy in patients with a prior negative biopsy: a consensus statement by AUA and SAR. J.Urol. 2016; 196: 1613-18

25. Venderlink W, van Luijtelaar A, Bomers JG et al. Results of targeted biopsy in men with magnetic resonance imaging lesions classified equivocal, likely or highly likely to be clinically significant prostate cancer. Eur. Urol. 2017; 73: 353-360

26. Bhat NR, Vetter JM, Andriole GL, Shetty AS, Ippolito JE, Kim EH. Magnetic resonance imaging-defined prostate-specific antigen density significantly improves the risk prediction for clinically significant prostate cancer on biopsy. J. Urol 2018; 126: 152-157

27. Distler FA, Radtke JP, Bonekamp D et al. The value of PSA density in combination with PI-RADS ${ }^{T M}$ for the accuracy of prostate cancer prediction. J.Urol 2017; 198: 575-582

28. Cuocolo R, Stanzione A, Rusconi G et al. PSA-density does not improve bi-parametric prostate MR detection of prostate cancer in a biopsy naïve population. Eur. J. Radiol. 2018; 104: 64-70

29. Thompson JE, Moses D, Shnier R et al. Multiparametric magnetic resonance imaging guided diagnostic biopsy detects significant prostate cancer and could reduce unnecessary biopsies and over detection: a prospective study. J.Urol. 2014; 192: 67-74

\section{Tables}

Due to technical limitations, tables $1,2,3$, and 4 are only available as downloads in the supplemental files section.

\section{Figures}


Fig. 1 ROC curves for combination of PI-RADS score and PSAD

All PCa

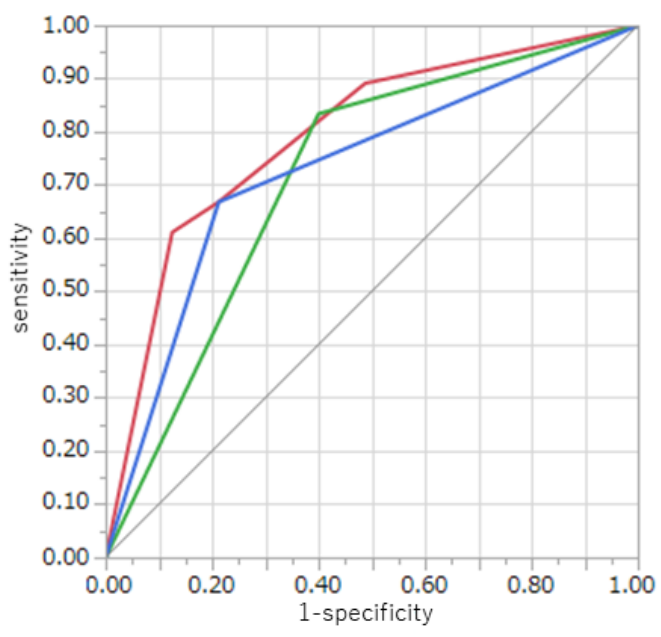

\begin{tabular}{l|c|} 
- PI-RADS score & $\mathrm{AUC}=0.717$ \\
- PSAD & $\mathrm{AUC}=0.728$ \\
\hline PSAD + PI-RADS score & $\mathrm{AUC}=0.793$ \\
\hline & $\mathrm{P}$ value \\
\hline Combined vs. PI-RADS & 0.0028 \\
\hline Combined vs. PSAD & $<0.0001$ \\
\hline PI-RADS vs. PSAD & 0.7709 \\
\hline
\end{tabular}

csPCa

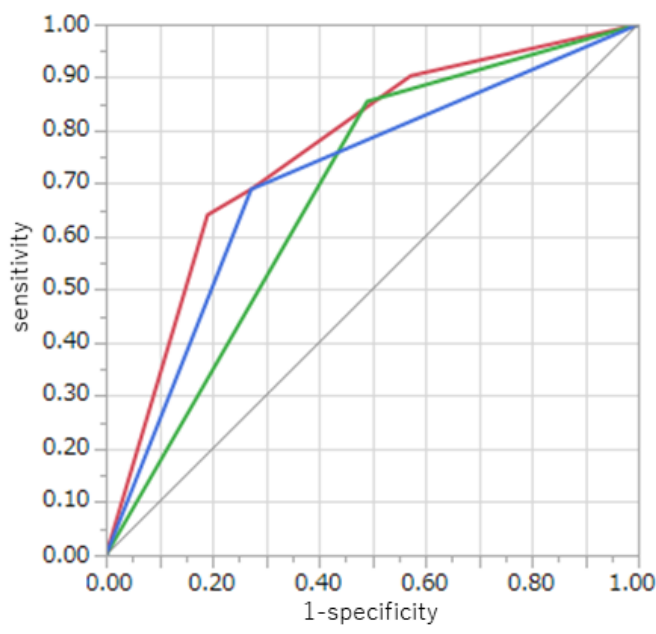

\begin{tabular}{l|c|} 
- PI-RADS score & $\mathrm{AUC}=0.682$ \\
- PSAD & $\mathrm{AUC}=0.709$ \\
\hline PSAD + PI-RADS score & $\mathrm{AUC}=0.761$ \\
\hline & $\mathrm{P}$ value \\
\hline Combined vs. PI-RADS & 0.0011 \\
\hline Combined vs. PSAD & $<0.0001$ \\
\hline PI-RADS vs. PSAD & 0.4422 \\
\hline
\end{tabular}

\section{Figure 1}

ROC curves for combination of PI-RADS score and PSAD The ROC curves for PI-RADS score, PSAD, and their combination were analyzed for their diagnostic value in the detection of both PCa and csPCa. Pairwise comparison of ROC curves were conducted with DeLong's test. For both PCaand csPCa, the combination of PI-RADS and PSAD showed a significantly higher AUC than each of the parameters alone. Abbreviations: ROC: receiver operating characteristic, PI-RADS: prostate reporting imaging and data system, PSAD: PSA density, PCa: prostate cancer, csPCa: clinically significant prostate cancer, AUC: area under curve 
Fig. 2 Biopsy results stratified by PI-RADS score

\section{Biopsy results by PI-RADS score}

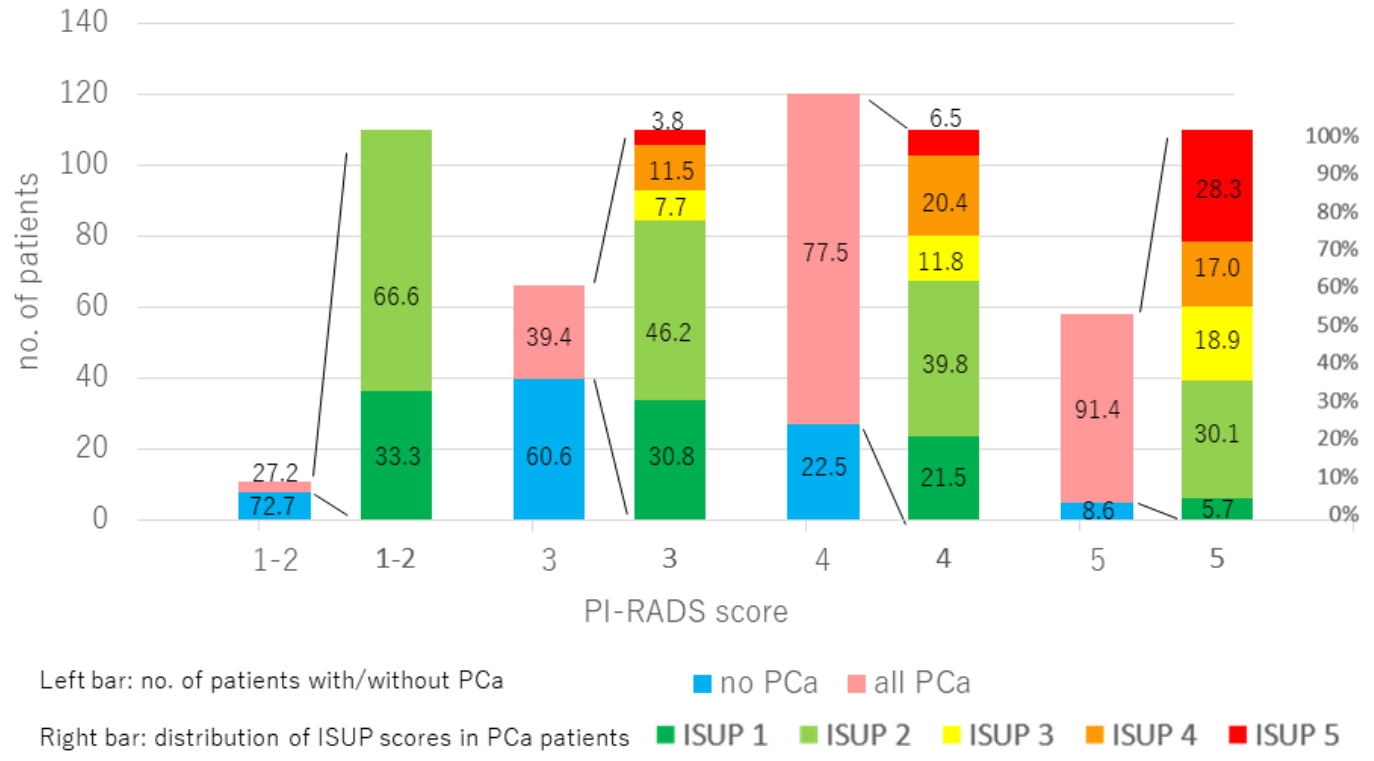

\section{Figure 2}

Biopsy results stratified by PI-RADS score Biopsy results for each PI-RADS score group were analyzed. Percentage of positive biopsies and ISUP score both showed a tendency to rise along with the PI-RADS score. All numerals represent percentages within the PI-RADS score group. Abbreviations: PI-RADS: prostate imaging reporting and data system, PCa: prostate cancer, ISUP: International society of urological pathology

\section{Supplementary Files}

This is a list of supplementary files associated with this preprint. Click to download.

- Table2.xlsx

- Table1.xlsx

- Table4.xlsx

- Table3.xlsx

- Suppl.Table.1.xlsx 